\title{
THE ISM IN NEARBY DWARF GALAXIES
}

\author{
L. M. YOUNG \\ Astronomy Department, University of Illinois \\ 1002 W. Green St., Urbana, IL 61801, USA \\ AND \\ K. Y. LO \\ Institute of Astronomy and Astrophysics, Academica Sinica \\ P. O. Box 1-87, Nankang, Taipei, Taiwan 11529, ROC
}

\section{Introduction}

In recent years, color-magnitude diagrams have revealed that nearby dwarf galaxies have a fascinating variety of star formation histories. The fact that the dwarfs have periods of star formation activity and inactivity raises several questions. How do the properties of the interstellar medium (ISM) in a galaxy affect its star formation rate? Are the interstellar media of dwarf galaxies similar to those in spirals? And what can we learn about dwarf galaxies' evolution from their gas contents?

We investigate these issues through observations of the neutral interstellar medium in a small sample of nearby dwarf galaxies. These include two dwarf irregulars (Leo A and Sag DIG), two dwarf ellipticals (NGC 185 and NGC 205), and two intermediate irregular/spheroidal galaxies (LGS 3 and the Phoenix dwarf), whose distances are $\approx 1$ Mpc or less. HI synthesis images of these galaxies probe their atomic media at resolutions of 60-160 pc. Observations of $\mathrm{CO}$ emission in the dwarf ellipticals clarify the relationship between atomic and molecular gas in those galaxies.

\section{Properties of the ISM}

HI in our own spiral Galaxy is made up of two phases, a cold phase and a warm phase. Four dwarf galaxies with current or recent star formation also show evidence of a cold atomic gas component. For example, the HI spectra in the irregulars Leo A and Sag DIG can be decomposed into highdispersion $(\sigma=8-10 \mathrm{~km} / \mathrm{s})$ and low-dispersion $(\sigma=3-5 \mathrm{~km} / \mathrm{s}$ ) components. We argue (Young \& Lo 1996a, 1997b) that these components are probably analogous to Galactic cold and warm neutral phases. Theoretical models of the HI medium can explain the presence of the two HI phases in the dwarf galaxies, even though the gas-phase metallicity and interstellar UV field are much lower in the dwarfs than in our own Galaxy. Twenty to thirty percent of the HI in those irregulars is in the cold component, with the remaining $\mathrm{HI}$ in the warm component; star formation seems to be associated with the cold HI component.

The dwarf elliptical galaxies NGC 185 and NGC 205 also show low-dispersion HI clumps associated with molecular clouds and dust patches (Young \& Lo 1996a, 1996b). LGS 3, the sample galaxy with the least amount of current star formation, shows only a high-dispersion warm HI phase. These results suggest that a cold atomic component is necessary for, or at least commonly associated with, star formation. The presence of a cold HI component should be a better diagnostic of conditions appropriate for star formation than measures of the total HI content. 


\section{Evolution of Elliptical and Spheroidal Galaxies}

The neutral gas contents of early-type galaxies are vital clues to understanding their evolution. For example, some giant elliptical galaxies do have significant HI, and the kinematics of this gas generally suggest that the gas was acquired in mergers or interactions (van Gorkom 1992). In contrast to the giant ellipticals, HI in the dwarf ellipticals NGC 185 and NGC 205 has a compact distribution and relatively low angular momentum (Young \& Lo 1997a). Thus, an internal origin for the gas in the dwarfs (stellar mass loss) is possible; mergers or interactions are not necessarily implicated.

The HI contents of the Local Group dwarf spheroidal galaxies also offer clues about their evolution. Current observations have shown very little HI in these galaxies (e.g. Knapp et al. 1978; Mould et al. 1990). It has been proposed that a strong burst of star formation activity might have expelled all of the ISM out of the galaxy (e.g. Dekel \& Silk 1986). The presence of gas in the intermediate irregular/spheroidal LGS 3 conflicts with this idea (Young \& Lo 1997b). Either LGS 3 has not had a burst of star formation activity, or the ISM is more difficult to expel than current models predict. We also detect two clouds of HI near the Phoenix dwarf spheroidal (Young \& Lo 1997b). However, the velocity of the galaxy is not known and we cannot be sure whether the HI clouds are associated with Phoenix or perhaps with the Magellanic Stream.

\section{References}

Dekel, A., \& Silk, J. 1986, ApJ, 303, 39

Knapp, G. R., Kerr, F. J., \& Bowers, P. F. 1978, AJ, 83, 360

Mould, J. R., Bothun, G. D., Hall, P. J., Staveley-Smith, L., \& Wright, A. E. 1990, ApJ, 362, L55

van Gorkom, J. 1992, in Morphological and Physical Classification of Galaxies, ed. G. Longo et al. (Dordrecht: Kluwer), p. 233

Young, L. M., \& Lo, K. Y. 1996a, ApJ, 462, 203

Young, L. M., \& Lo, K. Y. 1996b, ApJ, 464, L59

Young, L. M., \& Lo, K. Y. 1997a, ApJ, 476, 127

Young, L. M., \& Lo, K. Y. 1997b, ApJ, in press 